\title{
Correction to: Studies of the Biogeochemistry of Typical Estuaries and Bays in China
}

\author{
Zhiliang Shen
}

\section{Correction to:}

Z. Shen (ed.), Studies of the Biogeochemistry of Typical

Estuaries and Bays in China, Springer Earth System Sciences, https://doi.org/10.1007/978-3-662-58169-8

The book was inadvertently published with an incorrect affiliation "Institute of Oceanology, Chinese Academy of Sciences, Qingdao, China", whereas it should be "Center for Ocean Mega-Science, Chinese Academy of Sciences, Institute of Oceanology, Chinese Academy of Sciences, Qingdao, China”. The changes have been updated in the book. 